\title{
PD-L1 detection on circulating tumor-derived extracellular vesicles (T-EVs) from patients with lung cancer
}

\author{
Fei Wu ${ }^{1,2 \#}$, Yanzi Gu ${ }^{3 \#}$, Bin Kang ${ }^{1,2}$, Fabienne Heskia ${ }^{4}$, Alexandre Pachot ${ }^{5}$, Marc Bonneville ${ }^{6}$, Ping Wei ${ }^{7,8,9}$, \\ Ji Liang ${ }^{1,2}$
}

${ }^{1}$ Fudan University Shanghai Cancer Center-Institut Mérieux Laboratory, Cancer Institute, Fudan University Shanghai Cancer Center, Shanghai, China; ${ }^{2}$ bioMérieux (Shanghai) Company Limited, Shanghai, China; ${ }^{3}$ Biobank, Fudan University Shanghai Cancer Center, Shanghai, China; ${ }^{4}$ Global Medical Affairs, bioMérieux SA, Marcy l'Etoile, France; 'Open Innovation \& Partnerships Department, bioMérieux SA, Marcy l'Etoile, France; ${ }^{6}$ Institut Mérieux, Lyon, France; ${ }^{7}$ Department of Pathology, Fudan University Shanghai Cancer Center, Shanghai, China; ${ }^{8}$ Department of Oncology, Shanghai Medical College, Fudan University, Shanghai, China; ${ }^{9}$ Cancer Institute, Fudan University Shanghai Cancer Center, Shanghai, China

Contributions: (I) Conception and design: A Pachot, M Bonneville, P Wei, J Liang; (II) Administrative support: P Wei, J Liang; (III) Provision of study materials or patients: Y Gu, P Wei; (IV) Collection and assembly of data: F Wu, Y Gu; (V) Data analysis and interpretation: F Wu, B Kang, F Heskia, P Wei, J Liang; (VI) Manuscript writing: All authors; (VII) Final approval of manuscript: All authors.

"These authors contributed equally to this work.

Correspondence to: Ji Liang, PhD. bioMérieux (Shanghai) Company Limited, Shanghai 200032, China. Email: jill.liang@biomerieux.com; Ping Wei, PhD. Department of Pathology, Fudan University Shanghai Cancer Center and Department of Oncology, Shanghai Medical College, Fudan University, Shanghai 200032, China; Cancer Institute, Fudan University Shanghai Cancer Center, Shanghai, China. Email: weiping@fudan.edu.cn.

Background: Recent breakthroughs in therapies with immune checkpoint inhibitors (ICIs) have revolutionized the treatment of lung cancer. However, only $15-25 \%$ of patients respond to the ICIs therapy, and methods to identify those responsive patients are currently a hot research topic. PD-L1 expression measured on tumor tissues using immunohistochemistry (IHC) was approved as one of the companion diagnostic methods, but it is invasive and cannot be used to monitor dynamic changes in PD-L1 expression during treatments.

Methods: In this study, we developed an Epcam-PD-L1 extracellular vesicle (EV) detection prototype using the Simoa platform. This assay detected PD-L1 expression levels on tumor-derived exosomes from the lung cancer cell lines A549 and SK-MES1. In addition, 35 plasma samples from patients with lung cancer were tested with this assay and the results were compared to the tissue PD-L1 expression levels represented by the tumor proportion score (TPS).

Results: PD-L1 TPS-positive patients ( $\geq 1 \%$ IHC TPS) had significantly higher Simoa Epcam-PD-L1 signals than TPS-negative patients ( $<1 \%$ IHC TPS, P=0.026). The Simoa Epcam-PD-L1 area under curve (AUC) reached 0.776 , with a sensitivity of $92.86 \%$ and a specificity of $71.43 \%$. When PD-L1 TPS-positive patients were defined as having an IHC TPS $\geq 10 \%$, the greatest difference in Epcam-PD-L1 signals was observed between IHC TPS-positive and IHC TPS-negative groups ( $\mathrm{P}=0.0024)$ and the Simoa EpcamPD-L1 AUC reached 0.832. Finally, the Spearman's correlation coefficient showed a significant correlation between the TPS and Simoa Epcam-PD-L1 signals (0.428, P=0.0104).

Conclusions: Based on our results, our Simoa Epcam-PD-L1 EV detection assay is a potential liquid biopsy method to predict the PD-L1 expression level in patients with lung cancer.

Keywords: Programmed cell death ligand-1 (PD-L1); lung cancer; exosome; extracellular vesicle (EV)

Submitted Dec 17, 2020. Accepted for publication Apr 06, 2021.

doi: $10.21037 /$ tlcr-20-1277

View this article at: http://dx.doi.org/10.21037/tlcr-20-1277 


\section{Introduction}

Immunotherapies with immune checkpoint inhibitors (ICIs) have produced encouraging results in patients with non-small cell lung cancer (NSCLC) (1-6). Several antibodies against programmed cell death-1 (PD-1) and programmed cell death ligand-1 (PD-L1) have been approved as treatments in monotherapy or in combination with chemotherapy, other immunotherapies, and antiangiogenesis agents in many countries (7-10). The magnitude of the clinical benefit is generally associated with the tumor PD-L1 expression level $(1-5,11,12)$. One of the current approaches for the use of these therapeutic products is to stratify or select eligible patient populations by performing immunohistochemistry (IHC) with an anti-PD-L1 antibody on tumor tissues specimens. The expression of the PD-L1 protein is determined by calculating the tumor proportion score (TPS), which is the percentage of viable tumor cells showing partial or complete membrane staining at any intensity. The specimen is considered to express PD-L 1 if the TPS $\geq 1 \%$ and to express PD-L1 at high levels if the TPS $\geq 50 \%(1,13)$. Although this approach remains controversial $(14,15)$, patients with a higher PD-L1 TPS may obtain more benefits from ICIs therapies $(1,7,13,16-18)$.

Although PD-L1 IHC is widely used by clinicians to identify patients eligible for immunotherapy, it has several limitations. The limited availability of tumor tissues for testing, the variation driven by the biopsy site, and the variation in the reading of the assays by pathologists hamper the use of IHC as an ideal system for assessing PDL1 expression $(19,20)$. In addition, the regulation of PDL1 expression is a dynamic process that is unable to be adequately monitored by examining a tumor tissue biopsy. Thus, a less invasive, easier and more accurate technique to monitor tumor PD-L1 expression, such as the liquid biopsy, is urgently needed.

Tumor-derived extracellular vesicles (T-EVs), such as exosomes (30-150 nm) and microvesicles (150-1,000 $\mathrm{nm})$, whose molecular and genetic contents partially resemble the tumor cells from which they originate, are currently viewed as promising "liquid biopsies". T-EVs carry bioactive molecules and play diverse roles in tumor progression, including invasion, immune modulation, neovascularization, and metastasis (21-23). PD-L1 was reported to be expressed on the surface of EVs derived from tumor cell lines and detected in blood samples from patients with cancer (24-26). According to recent studies,
PD-L1 on EVs contributes to suppressing anti-tumor immunity and has the potential to serve as a biomarker in patients with cancer $(24,25,27)$. Additionally, some studies showed significant correlations between the levels of PDL1 in EVs and the pathological features of patients with cancer, such as the tumor size, stage or treatment response (24-26,28). However, the existing assays and technologies to detect $\mathrm{PD}-\mathrm{L} 1^{+} \mathrm{EVs}$ are either limited in sensitivity or highly complex, which prevent their use in medical practice. Thus, a method to rapidly detect and distinguish PD-L1 ${ }^{+}$EVs, particularly those derived from tumor cells, has yet to be developed.

Recently, using a single molecule array (Simoa), a highly sensitive immunoassay technology (29-32), we developed automatic EV/T-EV detection assays, enabling the direct profiling of EVs/T-EVs from the plasma of patients with cancer (33). In the current study, by capturing T-EVs with the same marker, epithelial cell adhesion molecule (Epcam), an important surface marker of epithelial tumor cell used as a biomarker of circulating tumor cells (CTCs) and T-EVs (34), we aim to develop a Simoa immunoassay prototype to detect $\mathrm{PD}-\mathrm{L} 1^{+} \mathrm{T}-\mathrm{EV}$ s from blood samples of patients with lung cancer and to compare PD-L1 expression in T-EVs and tumor tissues.

We present the following article in accordance with the MDAR reporting checklist (available at http://dx.doi. org/10.21037/tlcr-20-1277).

\section{Methods}

\section{Ethical statement}

The study was conducted in accordance with the Declaration of Helsinki (as revised in 2013). The study was approved by institutional review board of Fudan University Shanghai Cancer Center (No.: 050432-4-1911D) and individual consent for this retrospective analysis was waived.

\section{Prototype of the Simoa assay for detecting EVs}

Antibodies against Epcam and PD-L1 were selected and prepared for capture and detection according to the manufacturer's protocol (Quanterix, Lexington, MA, USA). Bead conjugation protocol was followed by an incubation at $4{ }^{\circ} \mathrm{C}$. The capture antibody concentration was adjusted to $0.2 \mathrm{mg} / \mathrm{mL}$ with Bead Conjugation Buffer and then paramagnetic carboxylated microparticles (Quanterix) were activated with $0.3 \mathrm{mg} / \mathrm{mL}$ 1-ethyl-3-(3- 
dimethylaminopropyl) carbodiimide hydrochloride (EDC) (Thermo Fisher Scientific, Waltham, MA, USA). Three microliters of the biotin solution (2 $\mathrm{mg}$ of NHS-PEG4Biotin dissolved in $383 \mu \mathrm{L}$ of $\mathrm{ddH}_{2} \mathrm{O}$ ) were added to $100 \mu \mathrm{L}$ of the detection antibody solution $(1.0 \mathrm{mg} / \mathrm{mL})$ for a molar biotinylation ratio of $40 \times$ to start the biotinylation reaction. The concentration of the recovered antibody was adjusted to $0.2 \mathrm{mg} / \mathrm{mL}$ and stored at $4{ }^{\circ} \mathrm{C}$.

In order to screen the best antibody pair for Simoa prototype, three monoclonal antibodies of PD-L1 were purchased from Abcam and Origene (Table S1). An antibody for Epcam (MAB9601, R\&D Systems) confirmed in a previously study (33) was taken as the capture antibody. Exosomes were collected from cell culture supernatant of HCT-116 for antibody pair testing due to its positive expression of Epcam and PD-L1 $(35,36)$. Finally, the antibody pair of MAB9601-TA507087 gave the highest signal/background ratio among all the antibodies tested, thus was selected for the further study (Figure S1).

\section{Simoa assay setup}

All Simoa measurements were performed using a fully automated Simoa HD-1 Analyzer (Quanterix). The microparticles coated with the Epcam capture antibody were diluted in Bead Diluent (Quanterix) to 500,000 microparticles per test. The PD-L1 detection antibody was diluted in Homebrew Detector/Sample Diluent (Quanterix) to a working concentration of $2 \mu \mathrm{g} / \mathrm{mL}$. The streptavidin$\beta$-galactosidase concentrate was diluted to a working concentration of $150 \mathrm{pM}$. The assay includes 3 steps. First, $25 \mu \mathrm{L}$ of the microparticle solution and $100 \mu \mathrm{L}$ of patient plasma (1:4 diluted) were incubated for 45 minutes in a reaction cuvette (Quanterix). Second, after several washes, the mixture and PD-L1 detection antibody were incubated for 5 minutes 15 seconds. Finally, $100 \mu \mathrm{L}$ of Streptavidin$\beta$-galactosidase (SBG) were added and incubated for 5 minutes 15 seconds. Resorufin $\beta$-D-galactopyranoside (RGP) was added after several washes and the sample was loaded into the array. The array was then sealed with oil and the microparticles were imaged. The automated analysis was performed using HD-1 Analyzer software (Quanterix). Simoa signal is expressed in average per bead (AEB) as previous described $(31,33)$. In short, AEB is determined by counting the number of wells containing both a bead and fluorescent signal ("on" well) relative to the total number of wells containing beads, using Poisson statistics and the digital or analog methods based on high or low concentrations of captured analyte. At low concentrations, the ratio of analytes to beads is small resulting in statistical distribution of individual molecules on the beads, giving Simoa its single molecule sensitivity.

\section{Cell culture and plasma}

Two lung cancer cell lines (A549, ATCC ${ }^{\circledR}$ CCL-185 ${ }^{\mathrm{TM}}$ and SK-MES1, ATCC ${ }^{\circledast}$ HTB-58 ${ }^{\mathrm{TM}}$ ) were cultured according to a standard protocol at Fudan University Shanghai Cancer Center-Institut Mérieux Laboratory. Cells were cultured in DMEM (L120KJ, YuanPei Biotech) supplemented with 10\% FBS (SH30084.03, HyClone) until the cells reached $80 \%$ culture confluence, and then washed twice with DMEM. After 36 hours of culture in DMEM, culture media were collected for direct testing or EV purification. For IFN $\gamma$ stimulation, cells were treated with $10 \mathrm{ng} / \mathrm{mL}$ IFN $\gamma$ (R\&D Systems) for 36 hours before collecting the culture media.

Clinical samples analyzed by Simoa platform were EDTA plasma samples collected from lung cancer patients before surgery. Three milliliter of patient whole blood were collected into the EDTA tube. Plasma was separated within 2 hours under 3,000 rpm centrifugation and transferred to the tissue bank of Fudan University Shanghai Cancer Center (FUSCC). The plasma samples used in this study were mainly collected in 2018 and stored at $-80^{\circ} \mathrm{C}$. Each participant signed an informed consent form allowing their samples to be used for research activities before they were enrolled in this study.

\section{Exosome/microvesicle purification}

Differential ultra-centrifugation was used to purify exosomes as described previously (37). Briefly, after collecting cell culture media, a low speed centrifugation (300 g, 10 minutes) step was applied to remove dead cells. Then, another centrifugation step at 2,000 $\mathrm{g}$ for 20 minutes was performed to remove cell debris. A subsequent 40 -minute centrifugation step at $10,000 \mathrm{~g}$ was applied to remove microvesicles. Exosomes were then collected by ultracentrifugation at $100,000 \mathrm{~g}$ for 2 hours using a Beckman Coulter Optima ${ }^{\mathrm{TM}}$ XP ultracentrifuge. Pellets were washed once with PBS and ulracentrifuged again at 100,000 $\mathrm{g}$ for 2 hours. The exosome concentration was calculated using a Flow NanoAnalyzer (NanoFCM Inc., Xiamen, China) according to the manufacturer's instructions. 


\section{Exosome flow cytometry}

An on-bead flow cytometry is used to detect exosomes/EVs $(38,39)$. In brief, one hundred microliters of the exosome solution were mixed with $10 \mu \mathrm{L}$ of $4 \mu \mathrm{m}$ aldehyde/sulfate latex beads (S37225, Thermo Fisher) for 15 minutes at room temperature with continuous rotation. After adding $1 \mathrm{~mL}$ of PBS, the mixture was incubated for 30 minutes at room temperature in rotation mode and centrifuged; then, the supernatant was removed. The reaction was stopped by incubating it with Stop Buffer (1 mM glycine, 2\% BSA, 1× PBS) for 30 minutes. After 3 washes, exosomes bound to beads were blocked with $1 \mathrm{~mL}$ of $10 \%$ BSA for 30 minutes. Following 3 washes, the beads were sequentially incubated with $1 \mu \mathrm{g}$ of the PD-L1 antibody (clone 28-8, ab205921, Abcam) and the HRP-Goat Anti-Rabbit IgG H\&L (ab97051) secondary antibody (1:20,000 dilution).

\section{Transmission electronic microscopy}

Electronic microscopy was performed at the Institute of Biochemistry and Cell Biology, Chinese Academy of Sciences (Shanghai). Freshly isolated exosomes were layered on copper grids with $0.125 \%$ Formvar in chloroform and stained with $1 \%$ uranyl acetate in $\mathrm{ddH}_{2} \mathrm{O}$. Immediately, exosomes on the grids were visualized using a JEOL JEM1011 transmission electron microscope (TESCAN).

\section{Analysis of PD-L1 protein expression using IHC}

IHC staining for the PD-L1 protein was performed on formalin-fixed paraffin-embedded (FFPE) tumor sections (4 $\mu \mathrm{m}$ thick) immediately after the operation, as described previously (40). In specimens with a maximum diameter greater than $1 \mathrm{~cm}$, a representative slide was selected for staining. The representative slide was defined as a section that contained the most diverse histological subtypes. This assay was performed on the Dako Autostainer Link 48 platform with an automated staining protocol using a mouse monoclonal anti-PD-L1 antibody (22C3). PD-L1 expression was evaluated by 2 board-certified pathologists in FUSCC, who were blinded to clinical data and patient outcomes, via calculating the TPS, which is defined as the percentage of PD-L1-positive tumor cells (TCs) relative to the total number of TCs. The evaluation of the score included partial or complete membranous staining (at least a $1+$ intensity). All other cells, such as tumor-associated immune cells, normal/non-neoplastic cells, and necrotic cells, were excluded from the evaluation. PD-L1 expression on TCs was classified into three levels: negative expression (TPS $<1 \%$ ), low expression (TPS 1-49\%), and high expression (TPS $\geq 50 \%$ ).

\section{Statistical analysis}

Plasma levels of circulating Epcam-PD-L1 EVs were compared with PD-L1 expression in solid tumor (TPS). A ROC curve was generated to calculate the area under curve (AUC), sensitivity and specificity of the Simoa EpcamPD-L1 assay in diagnosing PD-L1 positive patients. The highest AUC was determined by screening all possible TPS cutoffs. The tumor volume was calculated as the product of the three dimensions of the tumor in pathological records. Spearman's correlation coefficient between the Simoa Epcam-PD-L1 level, TPS and TPS multiplied by tumor volume were calculated to study their associations.

\section{Results}

\section{Design of the EV detection model}

The objective of this study was to detect PD-L1 expression levels on circulating tumor-derived EVs. Therefore, a Simoa EV detection assay was designed, as shown in Figure 1. In the Simoa disc array, over 20,000 wells were used in the signal collection step, with diameters of $4.5 \mu \mathrm{m}$ and depths of $3.25 \mu \mathrm{m}$. Considering the diameter of magnetic beads (2.7 $\mu \mathrm{m}$ diam.), the Simoa technology is capable of detecting most EVs, including exosomes $(30-150 \mathrm{~nm})$. First, a sandwich antibody complex is formed on microscopic beads. In samples containing EVs with Epcam expression, EVs are captured by Epcam capture antibodies on the magnetic beads. If EVs also express PD-L1, fluorophores are generated and fluorescence images are captured to calculate the signals. Several antibodies against Epcam and PD-L1 were tested based on the development of the homemade Simoa kit; Epcam (MAB9601, R\&D Systems) and PDL1 (TA507087, Origene) antibodies were selected for our Simoa prototype.

\section{Validation of the Epcam-PD-L1 Simoa assay}

Exosomes were isolated from two lung cancer cell lines, A549 and SK-MES1, using a previously described ultracentrifugation method to further validate the PD-L1 ${ }^{+}$ $\mathrm{EV}$ detection prototype (37). Isolated exosomes were further 


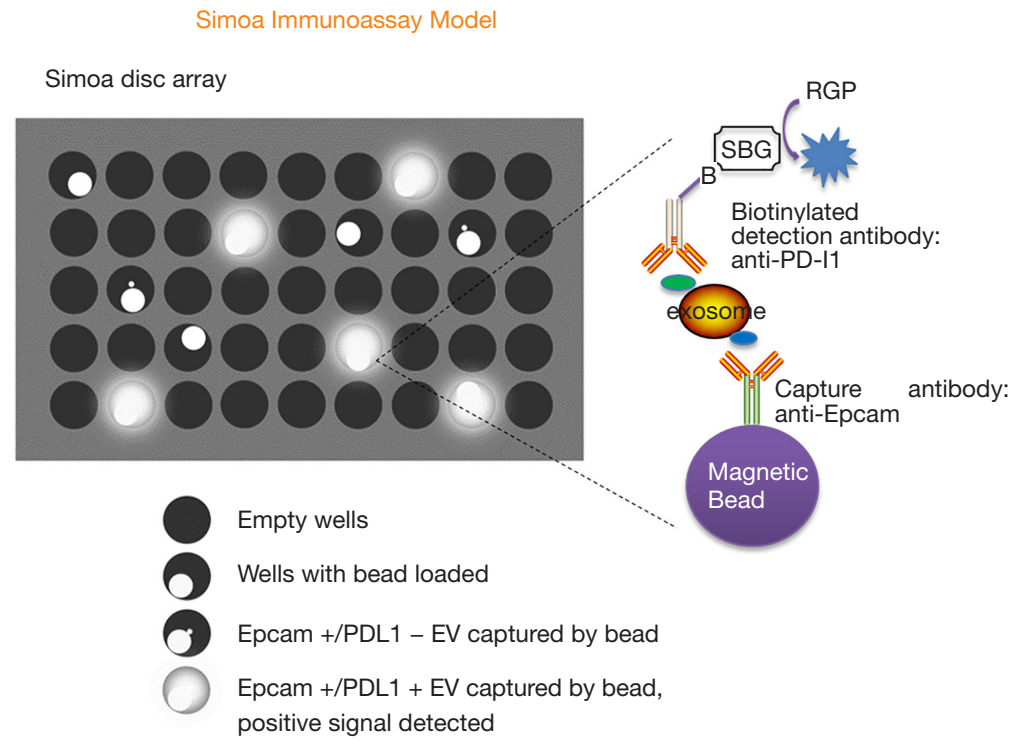

Figure 1 Prototype of the Simoa immunoassay for detecting EVs. Samples containing EVs are incubated with magnetic beads coated with capture Epcam antibodies. The bead-EV complexes are sequentially incubated with the biotinylated PD-L1 detection antibody and SBG, and then loaded onto the Simoa disc array. The catalytic reaction of SBG with RGP is restricted in the micro-well. The instrument detects an increasing fluorescent signal if a bead-EV-detector-SBG complex is loaded into the well. SBG, streptavidin- $\beta$-galactosidase; RGP, resorufin $\beta$-D-galactopyranoside.

analyzed using transmission electron microscopy (TEM) and showed the expected round morphology (Figure 2A). The concentration and size distribution of exosomes were analyzed using a Flow NanoAnalyzer (Figure 2B) (41). Based on the EV flow cytometry results, PD-L1 was almost undetectable on the surface of exosomes isolated from A549 cell line, while high PD-L1 expression was observed on the exosomes from the SK-MES1 cell line (Figure 2C, left panel). IFN $\gamma$ treatment has been reported to stimulate the upregulation of PD-L1 on the surface of tumor cells and EVs from various cancer cells $(21,22)$. After IFN $\gamma$ activation, $\mathrm{PD}-\mathrm{L} 1$ expression levels increased in exosomes from both cell lines (Figure 2C, right panel). Meanwhile, the same samples were analyzed using the Simoa PDL1-EV assay. Consistent with the flow cytometry results, Simoa testing showed higher signals in SK-MES1 cells than in A549 cells, and the IFN $\gamma$ treatment increased PD-L1 expression in both cell lines (Figure 2D). Thus, the Simoa Epcam-PD-L1 prototype may be a good assay to measure $\mathrm{PD}-\mathrm{L} 1^{+}$exosomes.

\section{Association between Simoa Epcam-PD-L1 level and the PD-L1 TPS}

We next tested Simoa Epcam-PD-L1 assay on plasma samples from 35 patients with lung cancer. Clinicopathological characteristics of the patients are summarized in Table 1. The median age of the patients was 64 years (range, $45-76$ years). Twenty-four of 35 (68.6\%) patients were male. More than half $(65.7 \%)$ of the patients had tumor smaller than $3 \mathrm{~cm}$ in largest dimension. Approximately 45.7\% (16/35) of the patients presented with lymph node metastasis. TNM stage showed that more T1 (42.9\%) and non-metastasis (91.4\%) patients were enrolled. Vascular invasion was observed in $65.7 \%(23 / 35)$ of patients. Clinical information for the TPS of PD-L1 IHC staining (22C3 PharmDx, Agilent Technologies, Santa Clara, CA, USA) was available for all patients. Twenty-eight patients had a TPS $\geq 1 \%$, which is the clinical accepted cutoff for a PD-L1-expressing patient (13).

We therefore compared the Simoa Epcam-PD-L1 levels between PD-L1-expressing and non-expressing patients using the aforementioned TPS cutoff (Figure $3 A$ ). When the TPS cutoff was set to $1 \%$, the Simoa Epcam-PD-L1 values were significantly higher in positive samples $(\mathrm{P}=0.026$, Figure $3 A$ ), with an AUC of 0.776 , a sensitivity of $92.86 \%$ and a specificity of $71.43 \%$ at the highest Youden index (Figure 3B). The highest AUC was observed when the TPS cutoff was set to $10 \%$, with 25 positive (TPS $\geq 10 \%$ ) and 10 negative (TPS $<10 \%$ ) samples. At this cutoff, the Simoa Epcam-PD-L1 level was significantly increased in positive 
A

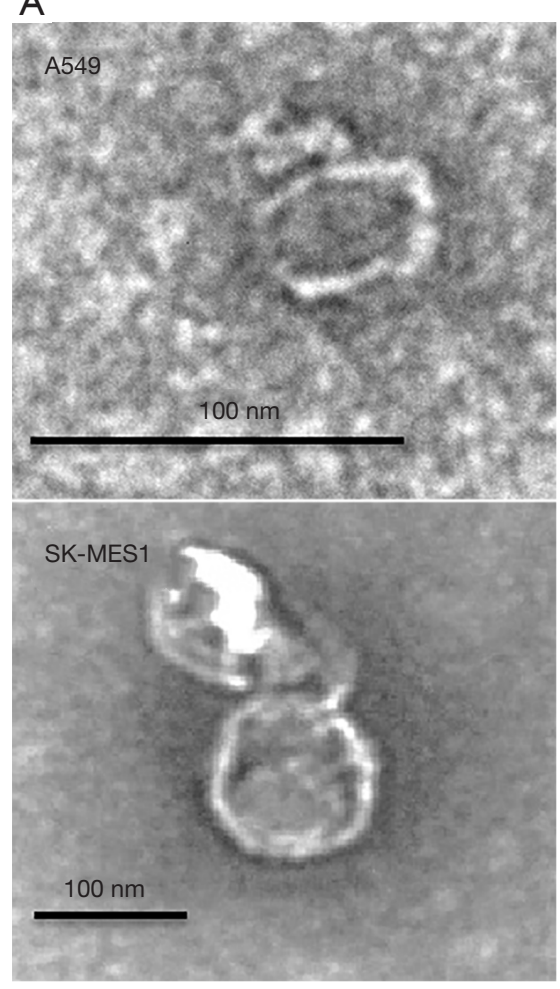

C
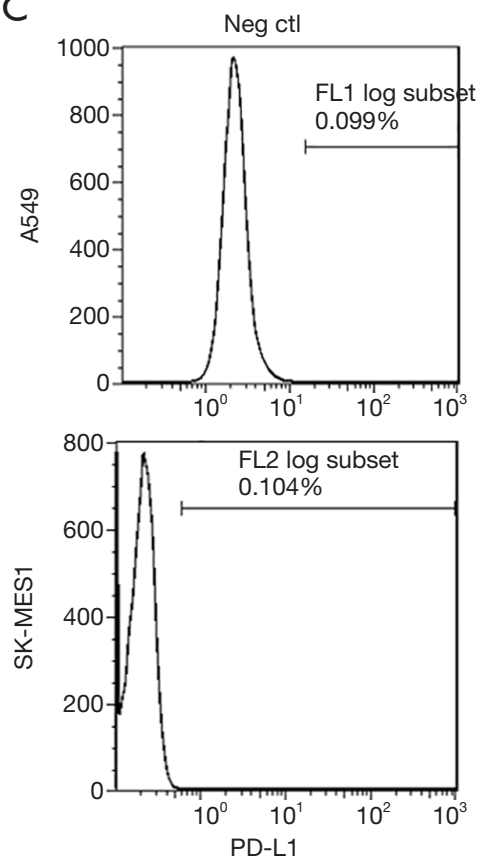

B
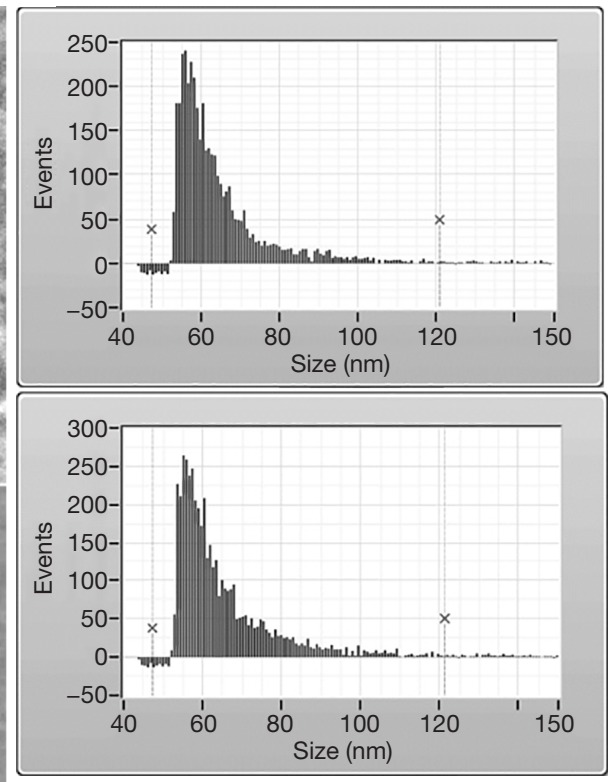

D

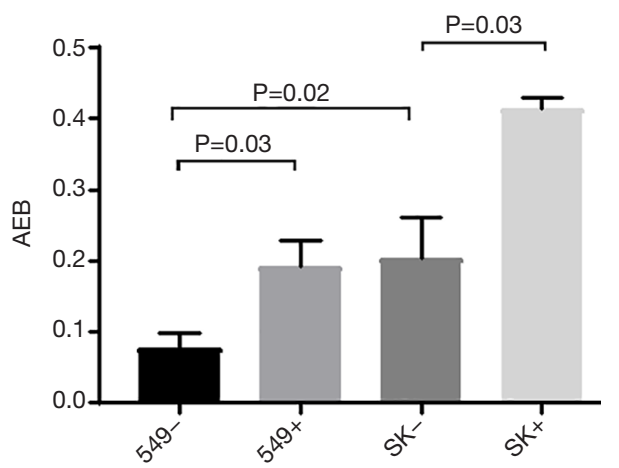

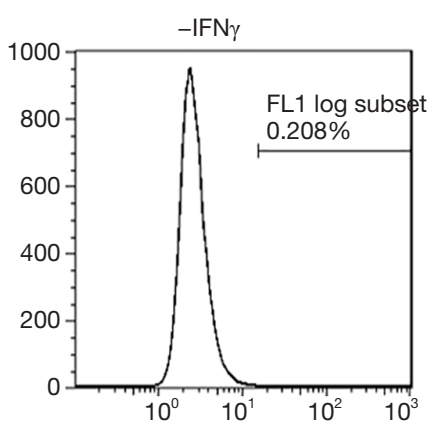

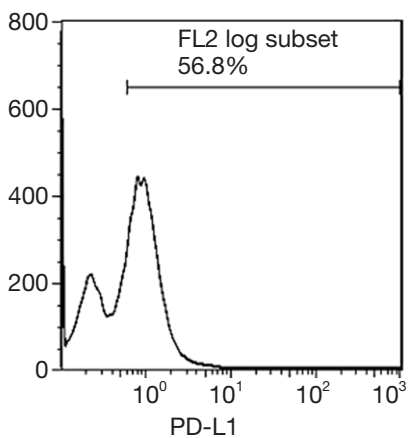

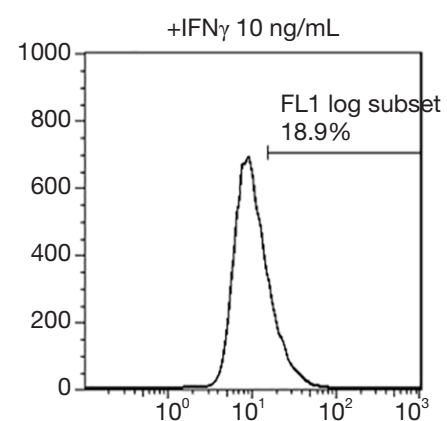

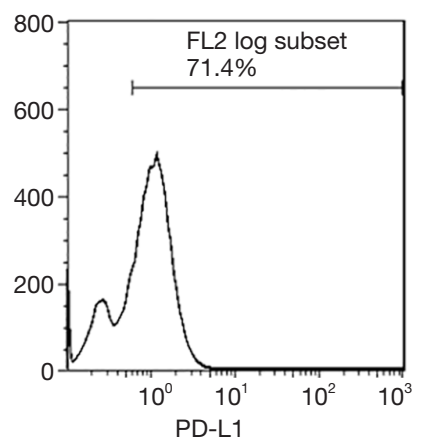

Figure 2 Validation of the Epcam-PD-L1 Simoa assay. (A) Exosomes isolated by ultracentrifugation from two lung cancer cell lines (A549 and SK-MES1) were analyzed using transmission electron microscopy and showed the typical exosomal morphology. (B) Samples showed the typical size distribution of exosomes when analyzed using a Flow NanoAnalyzer. Exosomal PD-L1 levels in SK-MES1 and A549 cells with and without IFN $\gamma$ treatment were evaluated using flow cytometry (C) and Epcam-PD-L1 Simoa assay (D). AEB, average enzyme per bead; Neg ctl, negative control; SK, SK-MES-1. 
Table 1 Clinicopathological features of patients with lung adenocarcinoma. Clinicopathological features of the patients, including age, sex, tumor size in the largest dimension, TNM stage, vascular invasion, and tumor proportion score of $\mathrm{PD}-\mathrm{L} 1$ expression

\begin{tabular}{|c|c|}
\hline Characteristic & No. (\%) \\
\hline Age, median [range] (years) & 64 [45-76] \\
\hline \multicolumn{2}{|l|}{ Sex } \\
\hline Male & $24(68.6)$ \\
\hline Female & $11(31.4)$ \\
\hline Tumor size, median [range] & $2.50[1.20-12.0]$ \\
\hline$\leq 3 \mathrm{~cm}$ & $23(65.7)$ \\
\hline$>3 \mathrm{~cm}$ & $10(28.6)$ \\
\hline \multicolumn{2}{|l|}{ T stage } \\
\hline T1 & $15(42.9)$ \\
\hline T2 & $5(14.3)$ \\
\hline T3 & $5(14.3)$ \\
\hline $\mathrm{T} 4$ & 8 (22.9) \\
\hline \multicolumn{2}{|l|}{ Lymph node } \\
\hline NO & $19(54.3)$ \\
\hline N1-3 & $16(45.7)$ \\
\hline \multicolumn{2}{|l|}{ Metastasis } \\
\hline MO & $32(91.4)$ \\
\hline M1 & $2(5.7)$ \\
\hline \multicolumn{2}{|l|}{ Vascular invasion } \\
\hline Negative & $11(31.4)$ \\
\hline Positive & $23(65.7)$ \\
\hline TPS, median [range] & $40 \%[0.5-98 \%]$ \\
\hline$\geq 1 \%$ & 28 \\
\hline$<1 \%$ & 7 \\
\hline
\end{tabular}

samples $(\mathrm{P}=0.0024$, Figure $3 C)$. The AUC reached 0.832, with both a sensitivity and specificity of $80 \%$ at the highest Youden index (Figure 3D). It has been demonstrated that TPS $\geq 50 \%$ is for PD-L1 high expression patients who would be response positive to ICIs treatment. We therefore also evaluated a discrimination of the Epcam-PD-L1 expression on these PD-L1 high expression patients. At $50 \%$ cutoff, Epcam-PD-L1 showed an increase in positive samples but not statistically significant $(\mathrm{P}=0.109)$, AUC was at 0.661 with sensitivity at $100 \%$ specificity at $36.84 \%$ at the highest Youden index (Figure S2).

In addition, we compared the Simoa results to the TPS score for each patient. Spearman's correlation coefficient for Simoa Epcam-PD-L1 and TPS was equal to 0.428 $(\mathrm{P}=0.0104,95 \%$ CI 0.110 to 0.666 , Figure $4 A$ ). A small increase in Spearman's correlation coefficient $(0.482$, $\mathrm{P}=0.003)$ was observed when the TPS was multiplied by the tumor volume (Figure 4B).

Based on our results, $\mathrm{PD}-\mathrm{L1}^{+} \mathrm{EV} /$ exosome levels measured using the Simoa Epcam-PD-L1 assay are significantly correlated with the TPS.

\section{Discussion}

Although still controversial, PD-L1 IHC is still one of the putative predictors of the response to PD-1/PD-L1-targeted checkpoint inhibitors. Since 2015, high expression of the PD$\mathrm{L} 1$ protein in tumor cells or tumor microenvironment (TME) has been identified to be a logical biomarker for predicting the efficacy of ICI therapy and was approved by the U.S. Food and Drug Administration as an indicator of initiating treatment for various solid tumors (15). On the other hand, the detection of PD-L1 expression through liquid biopsy, including tumor-derived soluble PD-L1 (sPD-L1) and PD-L1 on CTC or EVs/exosomes has garnered increasing attention to date. However, no evidence is available on whether circulating PD-L1 expression was consistent with tissue PD-L1 expression, and whether circulating PD-L1 levels had a similar value to predict the tumor response as tissue PD-L1 expression. In addition, the identification and quantification of biomarkers on EVs/exosomes in clinical samples remains challenging due to the complex isolation process. For example, to evaluate exosomes by the flow cytometry technology requires isolation of exosomes before detection, which is not feasible in clinical setting. Due to the small size of EVs/exosomes, an aldehyde/sulfate latex beads need to be used in the conventional flow cytometry to capture exosomes but in a non-specific way $(37,38)$. In contrast, Simoa platform might provide an ultrasensitive, non-invasive, fully automated, and high-throughput EV detection assay with double EVs biomarkers targeting (33). In the current study, for the first time, we developed a PD$\mathrm{L1}^{+} \mathrm{EV}$ detection assay based on the Simoa technology and identified a significant correlation of PD-L1 expression between T-EVs and tumor tissues. According to our results, our Simoa prototype might provide a non-invasive and dynamic method to monitor tumor PD-L1 expression in patients undergoing ICI therapy. 

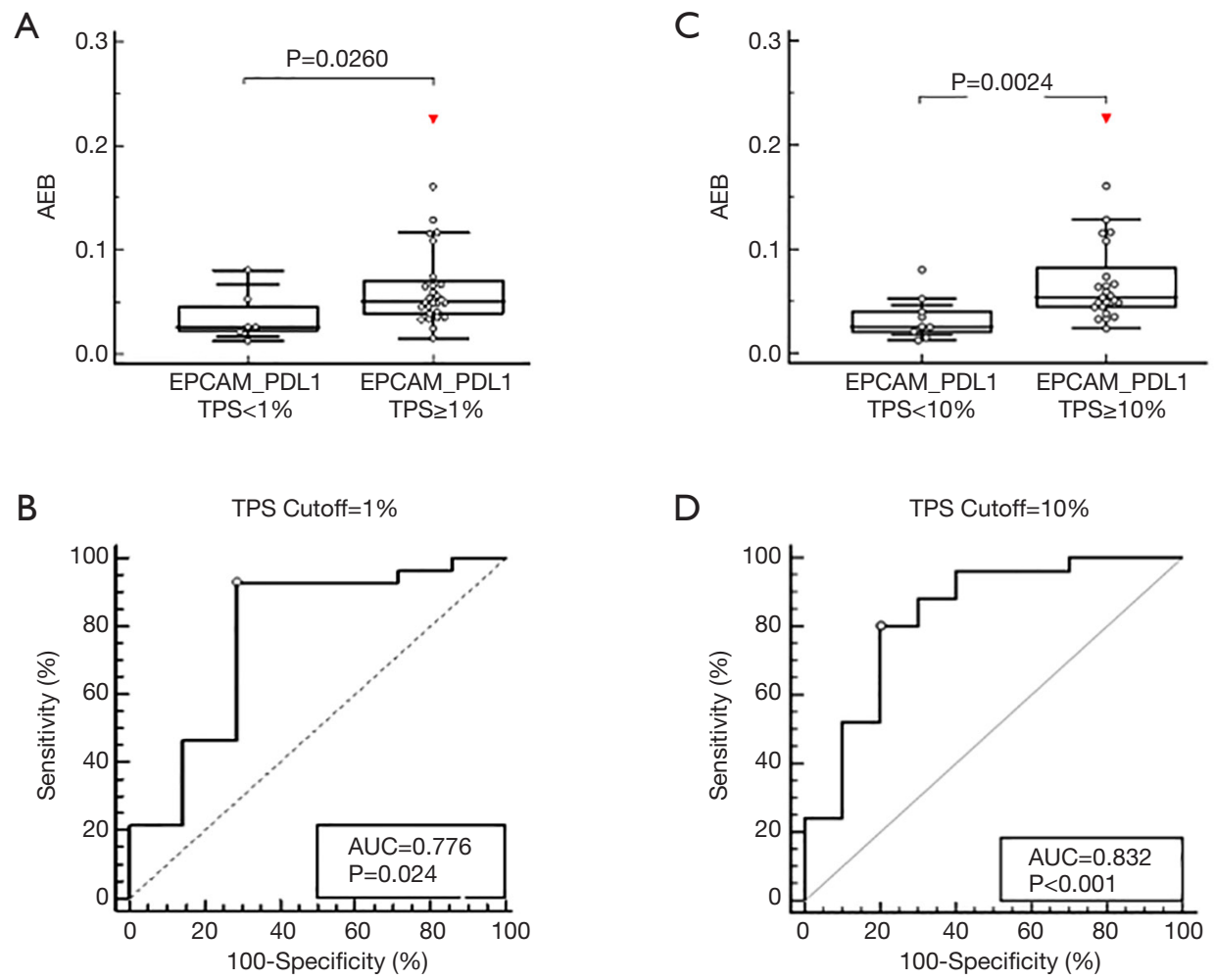

Figure 3 Comparison of Simoa Epcam-PD-L1 signals (AEB) in plasma samples from 35 patients with lung cancer and a positive or negative TPS. (A) At the $1 \%$ cutoff value, Simoa Epcam-PD-L1 signal (AEB) was significantly increased in samples from TPS-positive patients $(\mathrm{P}=0.026)$. (B) At the $1 \%$ cutoff value, the AUC reached 0.776 , with a sensitivity of $92.86 \%$ and a specificity of $71.43 \%$. (C) At the $10 \%$ cutoff value, the Simoa Epcam-PD-L1 signal (AEB) was significantly increased in TPS-positive samples (P=0.0024). (D) At the 10\% cutoff value, the best AUC was obtained at 0.832 , with a sensitivity of $80 \%$ and a specificity of $80 \%$. TPS, Tumor proportion score; AUC, area under curve.
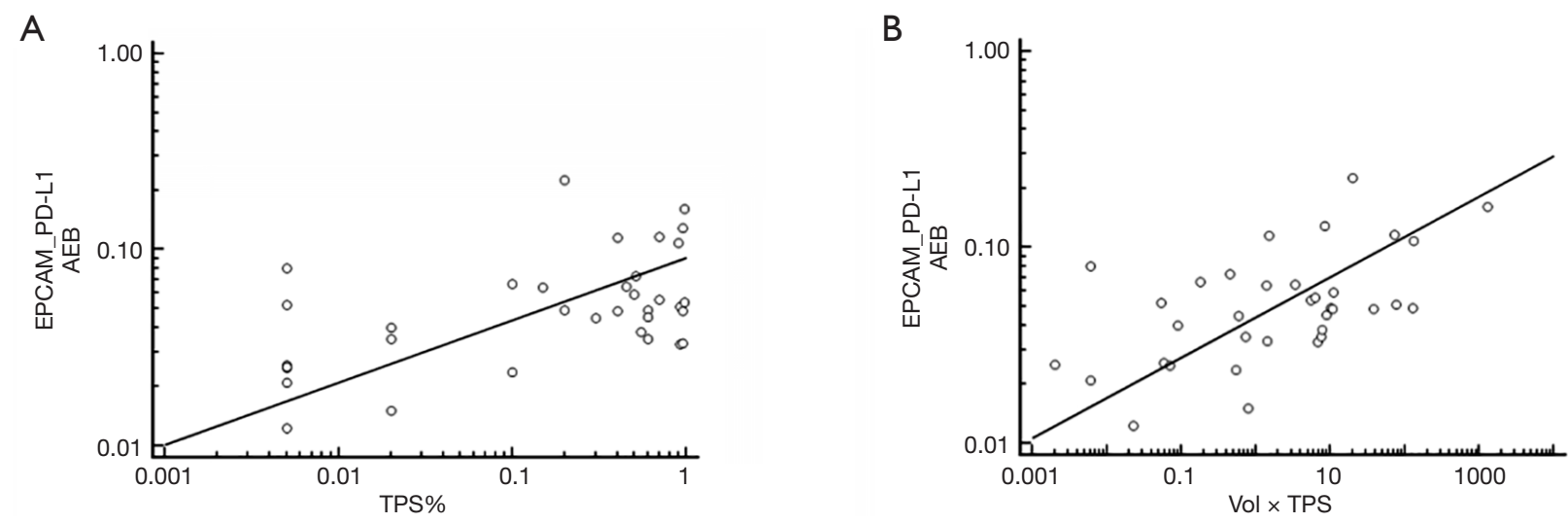

Figure 4 Spearman's correlation coefficients between Epcam-PD-L1 signals and TPS. (A) Spearman's correlation coefficient between the Epcam-PD-L1 signal and TPS is equal to 0.428 (P=0.0104, 95\% CI 0.110 to 0.666). (B) Spearman's correlation coefficient between the Epcam-PD-L1 signal and TPS is equal to $0.482(\mathrm{P}=0.003)$ when the TPS is multiplied by the tumor volume $($ Vol $\times$ TPS). 
In our study, plasma levels of PD-L1 ${ }^{+} \mathrm{T}-\mathrm{EV}$ s were significantly increased in PD-L1-positive patients (TPS $\geq 1 \%$ ) compared to PD-L1-negative patients (TPS $<1 \%$ ) $(\mathrm{P}=0.026)$. When the $10 \%$ threshold for the TPS was used to distinguish $\mathrm{PD}-\mathrm{L} 1$ positive patients, the best performance of the Epcam-PD-L1 assay was observed (AUC $=0.832$ ). At TPS cutoff set as $50 \%$, the performance of Epcam-PD-L1 assay decreased to $\mathrm{AUC}=0.661$. Based on those results, more PD-L1 IHC-positive patients also present high PD$\mathrm{L} 1$ signals on T-EVs, while relatively low levels of $\mathrm{PD}-\mathrm{L1}^{+}$ T-EVs are detected in PD-L1 IHC-negative patients. This characteristic might be useful in the future for monitoring therapeutic responses to ICIs. Moreover, the Simoa automatic exosome detection system potentially represents a nice technology to use in clinic practice.

Although the Simoa assay and TPS showed a similar trend in PD-L1 expression between PD-L1-positive and -negative patient groups, a subsequent analysis of Spearman's correlation coefficients between the results obtained with the two methods showed that the association between the PD-L1 ${ }^{+}$T-EV signal and PD-L1-IHC TPS results was still weak $(\mathrm{R}=0.428, \mathrm{P}=0.0104)$. We postulated that the discrepancy between the two methods might be explained by several factors. First, inter-tumor heterogeneity in PD-L1 expression is an important issue for an accurate TPS, while circulating T-EVs might theoretically provide a better representation of the whole tumor. The TPS defines the portion of tumor cells in the observed slide. Thus, the multiplication of the TPS by the tumor volume may be a good method to obtain an approximate number of tumor cells. Because we detected circulating exosomes in plasma with our Simoa Epcam-PD-L1 assay, this absolute tumor cell burden may be more relevant. Indeed, when TPS values are multiplied by the tumor volume, the correlation with the PD-L1 ${ }^{+}$T-EVs increased (Figure 4B, $\mathrm{R}=0.482, \mathrm{P}=0.003$ ).

Second, although Epcam may be the best biomarker for T-EVs and was chosen to capture T-EVs in our Simoa prototype, it is not expressed in $100 \%$ of carcinomas (33). High-level and mostly homogenous expression of Epcam were observed on $85 \%$ of adenocarcinomas and on $72 \%$ of squamous cell carcinomas (34). Finally, the wellknown PD-L1 IHC antibodies, such as 22C3 (Dako), 28-8 (Dako), SP142 (Ventana) and SP263 (Ventana), show different efficiencies for PD-L1 tissue staining and therefore different cutoffs for PD-L1-positive expression must be used (20). In our study, the PD-L1 TPS results were obtained with the $22 \mathrm{C} 3$ antibody, while an Origene antibody (TA507086) was chosen for the Simoa prototype due to its good performance. The differences in the efficiencies of the antibodies used in the two methods might also be responsible for the variation observed when comparing the results.

The encouraging results obtained with the Simoa PD$\mathrm{L} 1^{+} \mathrm{T}-\mathrm{EV}$ s assay were based on a population with a limited size. The current results must now be confirmed in a larger patient cohort. Additionally, other assays might be performed to obtain a better understanding of the technical issues raised above, including Epcam specificity and the different effects of anti-PD-L1 antibodies and finally to standardize procedures before clinical usage. At last, additional clinical trials should be conducted to determine whether PD-L1 expression on the circulating T-EVs has a similar value to tissue PD-L1 IHC in predicting the tumor response to ICI therapies and its expression cutoff sensitive to ICIs therapy should also be evaluated.

\section{Acknowledgments}

Funding: This work was supported by the National Science Foundation of China (grant numbers 81972185), Shanghai Natural Science Foundation (grant numbers 18ZR1407800), Rising-Star Program (grant number19QA1402200).

\section{Footnote}

Reporting Checklist: The authors have completed the MDAR reporting checklist. Available at http://dx.doi.org/10.21037/ tlcr-20-1277

Data Sharing Statement: Available at http://dx.doi. org/10.21037/tlcr-20-1277

Peer Review File: Available at http://dx.doi.org/10.21037/ tlcr-20-1277

Conflicts of Interest: All authors have completed the ICMJE uniform disclosure form (available at http://dx.doi. org/10.21037/tlcr-20-1277). PW reports that she has received research grants from National Science Foundation of China, Shanghai Natural Science Foundation of China, and Rising-Star Program of China. The other authors have no conflicts of interest to declare.

Ethical Statement: The authors are accountable for all aspects of the work in ensuring that questions related to the accuracy or integrity of any part of the work are 
appropriately investigated and resolved. The study was conducted in accordance with the Declaration of Helsinki (as revised in 2013). The study was approved by institutional review board of Fudan University Shanghai Cancer Center (No.: 050432-4-1911D) and individual consent for this retrospective analysis was waived.

Open Access Statement: This is an Open Access article distributed in accordance with the Creative Commons Attribution-NonCommercial-NoDerivs 4.0 International License (CC BY-NC-ND 4.0), which permits the noncommercial replication and distribution of the article with the strict proviso that no changes or edits are made and the original work is properly cited (including links to both the formal publication through the relevant DOI and the license). See: https://creativecommons.org/licenses/by-nc-nd/4.0/.

\section{References}

1. Herbst RS, Baas P, Kim DW, et al. Pembrolizumab versus docetaxel for previously treated, PD-L1-positive, advanced non-small-cell lung cancer (KEYNOTE-010): a randomised controlled trial. Lancet 2016;387:1540-50.

2. Petrylak DP, Powles T, Bellmunt J, et al. Atezolizumab (MPDL3280A) Monotherapy for Patients With Metastatic Urothelial Cancer: Long-term Outcomes From a Phase 1 Study. JAMA Oncol 2018;4:537-44.

3. Fehrenbacher L, Spira A, Ballinger M, et al. Atezolizumab versus docetaxel for patients with previously treated non-small-cell lung cancer (POPLAR): a multicentre, open-label, phase 2 randomised controlled trial. Lancet 2016;387:1837-46.

4. Borghaei H, Paz-Ares L, Horn L, et al. Nivolumab versus Docetaxel in Advanced Nonsquamous Non-Small-Cell Lung Cancer. N Engl J Med 2015;373:1627-39.

5. Rizvi NA, Mazieres J, Planchard D, et al. Activity and safety of nivolumab, an anti-PD-1 immune checkpoint inhibitor, for patients with advanced, refractory squamous non-small-cell lung cancer (CheckMate 063): a phase 2, single-arm trial. Lancet Oncol 2015;16:257-65.

6. Copur MD, Gauchan D, Ramaekers R. Durvalumab in Stage III Non-Small-Cell Lung Cancer. N Engl J Med 2018;378:868.

7. Gandhi L, Rodriguez-Abreu D, Gadgeel S, et al. Pembrolizumab plus Chemotherapy in Metastatic Non-Small-Cell Lung Cancer. N Engl J Med 2018;378:2078-92.

8. Brahmer JR, Lacchetti C, Schneider BJ, et al. Management of Immune-Related Adverse Events in Patients Treated With Immune Checkpoint Inhibitor Therapy: American Society of Clinical Oncology Clinical Practice Guideline. J Clin Oncol 2018;36:1714-68.

9. Haanen JB, Carbonnel F, Robert C, et al. Management of toxicities from immunotherapy: ESMO Clinical Practice Guidelines for diagnosis, treatment and follow-up. Ann Oncol 2017;28:iv119-42.

10. Haanen JB, Carbonnel F, Robert C, et al. Management of toxicities from immunotherapy: ESMO Clinical Practice Guidelines for diagnosis, treatment and follow-up. Ann Oncol 2018;29:iv264-6.

11. Herbst RS, Soria JC, Kowanetz M, et al. Predictive correlates of response to the anti-PD-L1 antibody MPDL3280A in cancer patients. Nature 2014;515:563-7.

12. Ibrahim R, Stewart R, Shalabi A. PD-L1 blockade for cancer treatment: MEDI4736. Semin Oncol 2015;42:474-83.

13. Mok TSK, Wu YL, Kudaba I, et al. Pembrolizumab versus chemotherapy for previously untreated, PD-L1expressing, locally advanced or metastatic non-small-cell lung cancer (KEYNOTE-042): a randomised, open-label, controlled, phase 3 trial. Lancet 2019;393:1819-30.

14. Patel SP, Kurzrock R. PD-L1 Expression as a Predictive Biomarker in Cancer Immunotherapy. Mol Cancer Ther 2015;14:847-56.

15. Sul J, Blumenthal GM, Jiang X, et al. FDA Approval Summary: Pembrolizumab for the Treatment of Patients With Metastatic Non-Small Cell Lung Cancer Whose Tumors Express Programmed Death-Ligand 1. Oncologist 2016;21:643-50.

16. Reck M, Rodriguez-Abreu D, Robinson AG, et al. Updated Analysis of KEYNOTE-024: Pembrolizumab Versus Platinum-Based Chemotherapy for Advanced NonSmall-Cell Lung Cancer With PD-L1 Tumor Proportion Score of 50\% or Greater. J Clin Oncol 2019;37:537-46.

17. Paz-Ares L, Luft A, Vicente D, et al. Pembrolizumab plus Chemotherapy for Squamous Non-Small-Cell Lung Cancer. N Engl J Med 2018;379:2040-51.

18. West H, McCleod M, Hussein M, et al. Atezolizumab in combination with carboplatin plus nab-paclitaxel chemotherapy compared with chemotherapy alone as firstline treatment for metastatic non-squamous non-smallcell lung cancer (IMpower130): a multicentre, randomised, open-label, phase 3 trial. Lancet Oncol 2019;20:924-37.

19. Remon J, Chaput N, Planchard D. Predictive biomarkers for programmed death-1/programmed death ligand immune checkpoint inhibitors in nonsmall cell lung 
cancer. Curr Opin Oncol 2016;28:122-9.

20. Hirsch FR, McElhinny A, Stanforth D, et al. PDL1 Immunohistochemistry Assays for Lung Cancer: Results from Phase 1 of the Blueprint PD-L1 IHC Assay Comparison Project. J Thorac Oncol 2017;12:208-22.

21. Kogure T, Yan IK, Lin WL, et al. Extracellular VesicleMediated Transfer of a Novel Long Noncoding RNA TUC339: A Mechanism of Intercellular Signaling in Human Hepatocellular Cancer. Genes Cancer 2013;4:261-72.

22. Atay S, Godwin AK. Tumor-derived exosomes: A message delivery system for tumor progression. Commun Integr Biol 2014;7:e28231.

23. Weston WW, Ganey T, Temple HT. The Relationship between Exosomes and Cancer: Implications for Diagnostics and Therapeutics. BioDrugs 2019;33:137-58.

24. Ricklefs FL, Alayo Q, Krenzlin H, et al. Immune evasion mediated by PD-L1 on glioblastoma-derived extracellular vesicles. Sci Adv 2018;4:eaar2766.

25. Chen G, Huang AC, Zhang W, et al. Exosomal PD-L1 contributes to immunosuppression and is associated with anti-PD-1 response. Nature 2018;560:382-6.

26. Theodoraki MN, Yerneni SS, Hoffmann TK, et al. Clinical Significance of PD-L1(+) Exosomes in Plasma of Head and Neck Cancer Patients. Clin Cancer Res 2018;24:896-905.

27. Poggio M, Hu T, Pai CC, et al. Suppression of Exosomal PD-L1 Induces Systemic Anti-tumor Immunity and Memory. Cell 2019;177:414-27.e13.

28. Ludwig S, Floros T, Theodoraki MN, et al. Suppression of Lymphocyte Functions by Plasma Exosomes Correlates with Disease Activity in Patients with Head and Neck Cancer. Clin Cancer Res 2017;23:4843-54.

29. Rissin DM, Fournier DR, Piech T, et al. Simultaneous detection of single molecules and singulated ensembles of molecules enables immunoassays with broad dynamic range. Anal Chem 2011;83:2279-85.

30. Wilson DH, Rissin DM, Kan CW, et al. The Simoa HD-1 Analyzer: A Novel Fully Automated Digital Immunoassay Analyzer with Single-Molecule Sensitivity and Multiplexing. J Lab Autom 2016;21:533-47.

31. Chang L, Rissin DM, Fournier DR, et al. Single molecule enzyme-linked immunosorbent assays: theoretical considerations. J Immunol Methods 2012;378:102-15.

32. Rivnak AJ, Rissin DM, Kan CW, et al. A fully-automated, six-plex single molecule immunoassay for measuring cytokines in blood. J Immunol Methods 2015;424:20-7.

33. Wei P, Wu F, Kang B, et al. Plasma extracellular vesicles detected by Single Molecule array technology as a liquid biopsy for colorectal cancer. J Extracell Vesicles 2020;9:1809765.

34. Baeuerle PA, Gires O. EpCAM (CD326) finding its role in cancer. Br J Cancer 2007;96:417-23.

35. Lu SH, Tsai WS, Chang YH, et al. Identifying cancer origin using circulating tumor cells. Cancer Biol Ther 2016;17:430-8.

36. Van Der Kraak L, Goel G, Ramanan K, et al. 5-Fluorouracil upregulates cell surface B7-H1 (PD-L1) expression in gastrointestinal cancers. J Immunother Cancer 2016;4:65.

37. Kowal J, Arras G, Colombo M, et al. Proteomic comparison defines novel markers to characterize heterogeneous populations of extracellular vesicle subtypes. Proc Natl Acad Sci U S A 2016;113:E968-77.

38. Bausero MA, Gastpar R, Multhoff G, et al. Alternative mechanism by which IFN-gamma enhances tumor recognition: active release of heat shock protein 72 . J Immunol 2005;175:2900-12.

39. Theodoraki MN, Hong CS, Donnenberg VS, et al. Evaluation of Exosome Proteins by on-Bead Flow Cytometry. Cytometry A 2021;99:372-81.

40. Zheng Q, Huang Y, Zeng X, et al. Correlation between PD-L1 Expression and Clinicopathological and Molecular Characteristics of Non-Small Cell Lung Cancer: A Large Scale Multi-Centric Real-World Study of Chinese Cohort. Laboratory Investigation 2020;100:1834-5.

41. Zhu S, Ma L, Wang S, et al. Light-scattering detection below the level of single fluorescent molecules for highresolution characterization of functional nanoparticles. ACS Nano 2014;8:10998-1006.
Cite this article as: Wu F, Gu Y, Kang B, Heskia F, Pachot A, Bonneville M, Wei P, Liang J. PD-L1 detection on circulating tumor-derived extracellular vesicles (T-EVs) from patients with lung cancer. Transl Lung Cancer Res 2021;10(6):2441-2451. doi: $10.21037 /$ tlcr-20-1277 
Supplementary

Table S1 Tested PD-L1 antibody

\begin{tabular}{lccl}
\hline Name & Company & Catalogue number & Source \\
\hline PD-L1-1 & Abcam & ab205921 & Monoclonal Rabbit IgG Clone \# 28-8 \\
PD-L1-2 & Origene & TA507087 & Monoclonal Mouse IgG1 Clone \#OTI2C7 \\
PD-L1-3 & Origene & TA808771 & Monoclonal Mouse IgG1 Clone \#OTI2C7 \\
\hline
\end{tabular}

Three monoclonal antibodies of PD-L1 were purchased from Abcam and Origene. Their performance in T-EV detection was further evaluated together with anti-Epcam antibody (MAB9601, R\&D Systems).

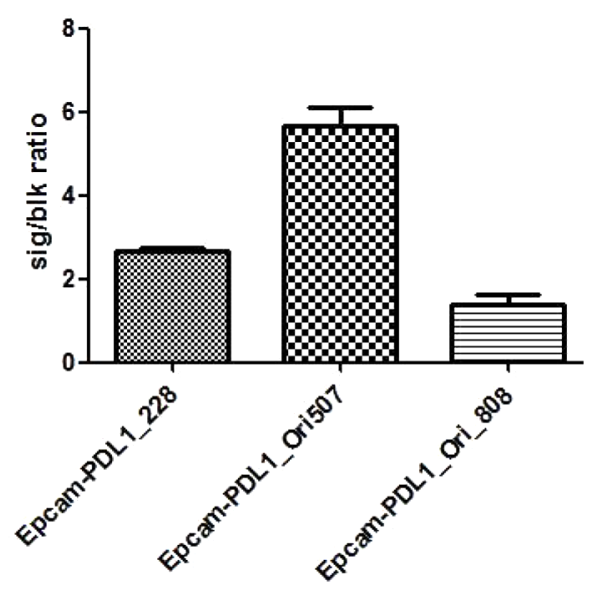

Figure S1 Performance of the three antibody pairs in detection of purified exosomes. Exosomes were purified from supernatants of HCT-116 cell culture and were used to evaluate the best EpcamPD-L1 antibody pair on the Simoa platform. sig/blk ratio: signal vs background ratio.
A

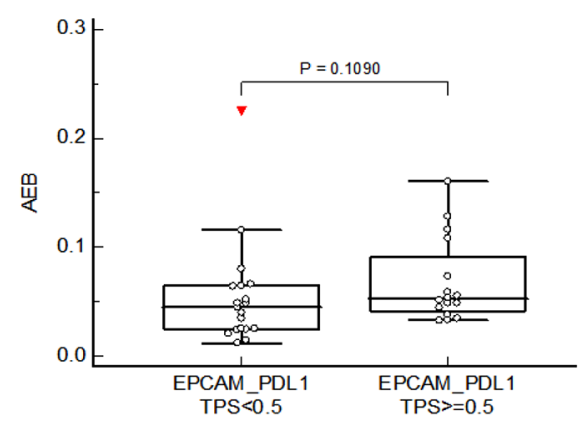

B

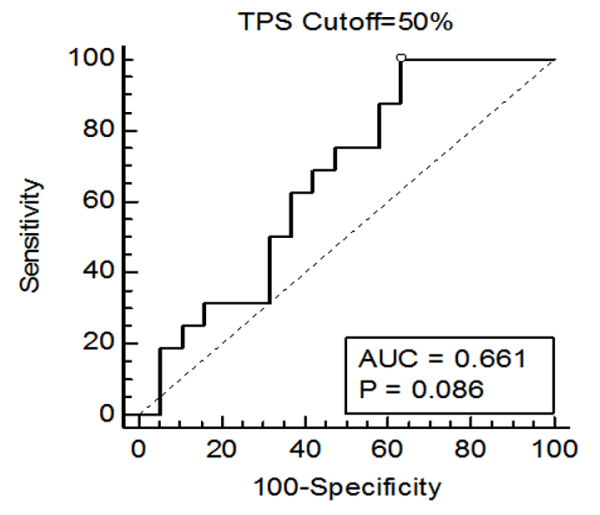

Figure S2 Comparison of Simoa Epcam-PD-L1 signals (AEB) in plasma samples from 35 patients with lung cancer and a high or low TPS. (A) At the 50\% cutoff value, TPS-high patients had higher but not significant value than TPS-low patients ( $\mathrm{P}=0.109)$. (B) The best AUC was obtained at 0.661 , with a sensitivity of $100 \%$ and a specificity of $36.84 \%$. 